\title{
When Far Becomes Near: Remapping of Space by Tool Use
}

\author{
Anna Berti \\ Università di Torino, Italy \\ Francesca Frassinetti
}

Università di Bologna, Italy

\begin{abstract}
- Far (extrapersonal) and near (peripersonal) spaces are behaviorally defined as the space outside the hand-reaching distance and the space within the hand-reaching distance. Animal and human studies have confirmed this distinction, showing that space is not homogeneously represented in the brain. In this paper we demonstrate that the coding of space as "far" and "near" is not only determined by the hand-reaching distance, but it is also dependent on how the brain represents the extension of the body space. We will show that when the cerebral representation of body space is extended to include objects or tools used by the subject, space previously mapped
\end{abstract}

as far can be remapped as near. Patient P.P., after a right hemisphere stroke, showed a dissociation between near and far spaces in the manifestation of neglect. Indeed, in a line bisection task, neglect was apparent in near space, but not in far space when bisection in the far space was performed with a projection lightpen. However, when in the far space bisection was performed with a stick, used by the patient to reach the line, neglect appeared and was as severe as neglect in the near space. An artificial extension of the patient's body (the stick) caused a remapping of far space as near space.

\section{INTRODUCTION}

One of the more central issue in cognitive neuroscience is how the brain constructs a map of the external world and how this map interacts with the representation of our body, in order to be able to deal with objects placed in the surrounding space.

We can detect, locate, orient to, and reach for an object. All these operations can be distinguished according to the sector of space in which they occur and to the action needed for accomplishing the task (Colby \& Duhamel, 1996; Rizzolatti, Riggio, \& Sheliga, 1994; Brouchon, Joanette, \& Samso, 1986). For instance, if the object of interest is located in the immediate surroundings of the body (peripersonal space), manual reaching and grasping can be achieved without locomotion. On the contrary, if the object of interest is placed outside a direct manual reaching (extrapersonal space) locomotion is needed for subsequent action to the object. Alternatively, the subject can use a tool to reach and grasp for far objects. In any case, we need to encode the position of the objects with respect to the position of the body and body parts. How is the location of objects placed in different sectors of space coded by the brain?

In animal studies there are reports that show that far and near space are separately represented in the brain. Area 8 of the monkey frontal lobe has different kinds of

neurons related, among other things, to the coding of saccades (e.g., Bruce \& Goldberg, 1985; Goldberg \& Bushnell, 1981). Although the simple coding of saccades may not be equivalent to the analysis of far space, some authors have pointed to the possibility that an area involved in eye movement programming might contribute to far space representation. Therefore, based on the physiological properties of area 8 neurons and on ablation studies showing that lesions of monkeys' frontal eye-fields causes inattention for stimuli presented contralateral to the brain damage (Latto \& Cowey, 1971) especially in the far space (Rizzolatti, Gentilucci, \& Matelli, 1985), Rizzolatti and Gallese (1988) proposed that area 8 is involved in far space representation. Furthermore, Colby, Duhamel, and Goldberg (1996) showed that neurons in area LIP (that is richly connected with, and physiologically similar to, area 8) might be another neural substrate for the representation of far space in monkeys. Area LIP neurons have independent sensory and motor responses and saccade-related burst. Interestingly, they discharge before a saccade even when there has been no recent visual stimulus and they are strongly responsive in a task in which the saccades are expressively forbidden. Moreover, attention to spatial location modulates the sensory response to the onset of the stimulus. Altogether, the physiological properties of 
Table 1. Means of the Absolute Displacement Errors and of Percentages of Neglect Displacement for Each Condition of the Experiment

\begin{tabular}{llll}
\hline Space & Modality & Short lines & Long lines \\
\hline Near & reaching & $24.8(24.8 \%)$ & $66.7(33.3 \%)$ \\
Near & pointing & $22.1(22.1 \%)$ & $51.6(25.8 \%)$ \\
Far & reaching & $58.5(29.2 \%)$ & $96.4(24.1 \%)$ \\
Far & pointing & $17.1(8.5 \%)$ & $39.1(9.7 \%)$ \\
\hline
\end{tabular}

these neurons suggest that they cannot be regarded exclusively as analyzing visual stimuli or as exclusively planning saccades. Because the common factor underlying the activation of these neurons appeared to be the location of an event in space, they have been considered the ideal candidates for far space representation.

On the contrary, near space seems to be represented in frontal area 6 and in the rostral part of the inferior parietal lobe, e.g., in area $7 \mathrm{~b}$ (Leinonen, Hyvarinen, Nymani, \& Linnankoski, 1979) and in area VIP (Duhamel, Bremmer, BenHamed, \& Graf, 1997; Colby, Duhamel, \& Goldberg, 1993). Some VIP neurons seem to code an ultra-near space, centered around the mouth. These neurons are most sensitive when a static stimulus is presented very close $(5 \mathrm{~cm})$ to the animal head. In these areas, peripersonal space is coded in strict relation to the body. Some neurons code for the position of a tactile stimulus delivered to the animal skin and for a visual stimulus presented in the space near the part of the body where the tactile field is located (bimodal neurons, Fogassi et al., 1996; Graziano, Yap, \& Gross, 1994; Graziano \& Gross, 1995; Gentilucci et al., 1988). Monkeys with lesions in these areas show inattention for stimuli located near the body whereas far stimuli are detected (Rizzolatti, Matelli, \& Pavesi, 1983). Following these studies, area 8 and parietal area LIP (Thier \& Andersen, 1997) have been considered as the anatomical correlate for far space coding, whereas area 6 and the rostral part of the inferior parietal lobe have been considered as the anatomical correlate for near space coding (Rizzolatti \& Gallese, 1988).

Recent neuropsychological investigations seem to suggest that in humans, space is coded in a way similar to monkeys. For instance, evidence in favor of the existence of a visuo-tactile peripersonal space has been recently offered by studies on cross-modal extinction. These studies showed that visual stimuli delivered in the near-peripersonal (but not in the far extrapersonal) space can extinguish tactile stimuli delivered controlaterally, on the corresponding sector of the body, e.g., the hand or the face (hand: Làdavas, di Pellegrino, Fernè, \& Zeloni, 1998; di Pellegrino, Làdavas, \& Farnè, 1997; face: Làdavas, Zeloni, \& Farnè, 1999).

A single case study by Halligan and Marshall (1991) showed that a dissociation between near and far space coding could also be suggested for humans. Their patient, following a right-hemisphere stroke, showed marked left visual-spatial neglect on a line-bisection task in near space that was reduced when the task was carried out in far space. Cowey, Small, and Ellis (1994) and Vuilleumier, Valenza, Mayer, Reverdin, and Landis (1998) reported the opposite dissociation: more severe neglect in far space than in near space. Thus, based on these studies, one may conclude that the distinction between near and far space is not simply descriptive, but that the brain has different ways for coding the position of objects placed in different location with respect to body coordinates (see Shelton, Bowers, \& Heilman, 1990 for further dissociations within the near space). If the brain constructs different maps according to far and near space, a question we can ask is whether "far" and "near" are derived simply referring to the reaching distance or whether coding of spatial positions is a more dynamic operation that can be influenced by using tools that modify the spatial relation between the body and the object. Iriki, Tanaka, and Iwamura (1996) found in the monkey parietal lobe bimodal neurons that coded the schema of the hand, similar to those studied by Rizzolatti et al. (Fogassi et al., 1996; Gentilucci et al., 1988) and by Graziano et al. (1994). These neurons fired when a tactile stimulus was delivered to the monkey's hand and when visual objects were presented near the hand tactile receptive field. The most striking characteristic of these neurons was that their visual receptive field was modified, during a reaching movement performed with a rake, to include the entire length of the rake and to cover the expanded accessible space. In other words, in that experiment, the body schema was altered using the tool (Head \& Holmes, 1911): The tool was assimilated to the hand, becoming part of the hand representation (Aglioti, Smania, Manfredi, \& Berlucchi, 1996; Paillard, 1993).

A possible consequence of the modification of the spatial relations between the body and the object caused by using a tool that extends the reaching space might be that far is remapped as near.

In the present study, we explored this possibility in a patient, P.P., who, after damage to the right hemisphere, was affected by visual neglect, a disturbance that impairs the processing and the exploration of the space contralateral to the brain lesion. P.P. showed neglect in a line-bisection test in near space, but not in far space (see

Table 2. Means of the Percentages of Rightward Displacement in Near and Far Space in the Reaching and Pointing Modality, Irrespective of Line Length (Interaction Space $\times$ Modality)

\begin{tabular}{lcc} 
& Reaching & Pointing \\
\hline Near & 29.1 & 24 \\
Far & 26.7 & 9.2 \\
\hline
\end{tabular}


Methods). Our prediction stated that if the bisection of lines in far space was executed with a tool (a stick) that extended the representation of the body to include the space reachable by the tool, then far space should have been treated as near space, and therefore should have become affected by neglect. Based on these assumptions, the patient was asked to bisect short and long lines, in near and far space, by pointing to the lines with a projection lightpen or by reaching them with the index finger or with a stick.

\section{RESULTS}

Displacement errors were calculated in millimeters. Displacement of the subjective midpoint to the right of the objective midpoint of the line is indicated with the sign "+" whereas the sign "- " indicates leftward displacement. The absolute displacement errors and the means of the percentages of the displacement errors for each condition are reported in Table 1 .

A repeated $2 \times 2 \times 2$ measure ANOVA was performed on the arcsine transformed percentages of displacement. We set the significant level at $p=.01$. Space (Near and Far), Line Length (Short and Long), and Bisection Modality (Reaching and Pointing) were the main factors. The analysis showed that Space $[F(1,19)=43.27, p<.00001]$, Bisection Modality $[F(1,19)=55.98, p<.000001]$ and the interaction Space $\times$ Modality $[F(1,19)=29.11, p<.0001]$ were significant sources of variance. Indeed, rightward displacement was greater in near than in far space $(26.5 \%$ vs. $18 \%$ ). Moreover, the main effect of Modality showed that the rightward displacement was greater in the reaching $(27.8 \%)$ than in the pointing modality (16.6\%). However, the interaction Space $\times$ Modality (see Table 2) showed that this was only true in the far space, where the patient's displacement to the right was $26.7 \%$ when she was reaching with the stick, and

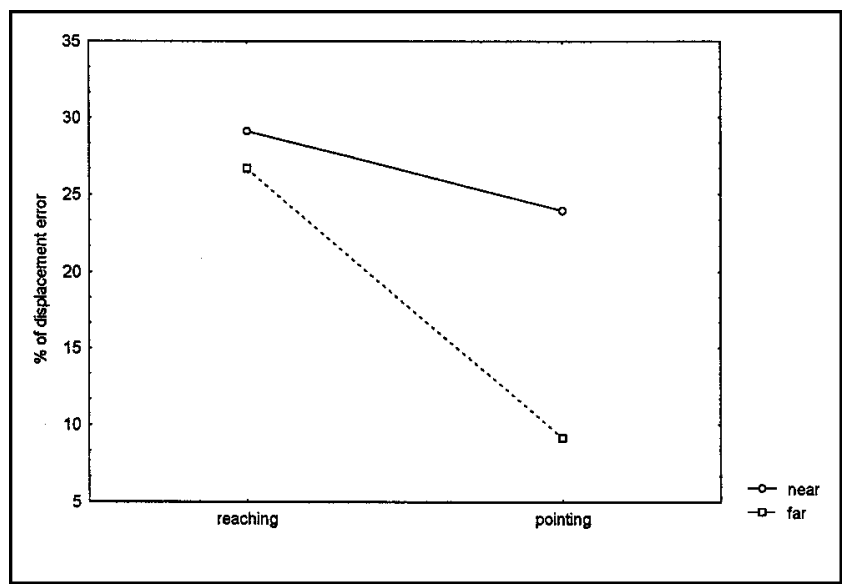

Figure 1. Percentages of rightward displacement as a function of space and modality. only 9\% when she was using the lightpen (NeumanKeuls post hoc comparison: $p<.000001)$. On the contrary, as shown in Figure 1, in near space no difference related to bisection modality was found (reaching with the stick $=29.1 \%$, pointing $=24 \%$ ).

The interaction Space $\times$ Length was also significant $[F(1,19)=20.58 p<.001]$. Post hoc comparison showed that rightward displacement in near space was greater for long (29.6\%) than for short lines (23.5\%), $p<.001$, whereas in far space this difference was not observed. Within far space, the difference between long (24.1\%) and short (29\%) lines in the reaching modality, was not statistically significant.

\section{DISCUSSION}

This data firstly shows that patient P.P. had neglect in near space, but not in far space, when she had to bisect a line using a lightpen. Therefore, our study provides a confirmation that impaired coding of near space can coexist with good coding of far space, as was first described by Halligan and Marshall (1991). Vuilleumier et al. (1998), and Cowey et al. (1994) described the opposite dissociation, i.e., neglect for far, but not for near, space. These double dissociations have been considered an indirect demonstration that the neural representation of the space outside the hand reaching and of the space within the hand reaching involves different brain mechanisms and, possibly different brain areas that are alternatively activated on the basis of the hand-reaching distance. Recently, Cowey, Small, and Ellis (1999), studying line bisection at different distances from the body, have shown that in patients with neglect for far, but not for near space, there was no sudden change in the patient's performance going from near to far distances. This might suggest that coding of far and near space does not depend on the switch between different neural maps, coding different space sectors. However, Cowey et al. have pointed out that their results are not incompatible with the existence of discrete brain mechanisms for space representation. They argue that the gradient effect, when observed, may be related to the great number of frontal and parietal cortical visual areas whose neurons are sensitive to the distance of the visual stimuli. As a result, if a lesion affects a region chiefly or exclusively concerned with near or far space, there will be ample opportunities for compensation (Cowey et al., 1999). Therefore, on the basis of these arguments, we may advance the hypothesis that also in our patient a discrete mechanism for far space coding was still working, whereas a separated mechanism of near space coding was damaged by the lesion.

Interestingly, although Halligan and Marshall (1991) did not present a reconstruction of the patient's cerebral damage, the lesion they described seems to be very similar to that of patient P.P. Both patients 
had a large infarct of the lateral aspect of the right hemisphere with some sparing of the frontal cortex. Therefore, one can advance the hypothesis that the antero-medial aspect of the right frontal lobe (e.g., the supplementary eye field) can be sufficient for far space coding. This hypothesis is based on the assumption that brain areas involved in motor programming are also involved in spatial representation, as discussed in the Introduction. In any case, it is important to remember that the lesions, both in Halligan and Marshall's case and in our case, were so large that drawing firm conclusions from the available anatomical data is difficult.

In our experiment we only used a line bisection task, so we cannot say whether the dissociation found in neglect between near and far space would be found also with other kind of stimuli. The only study that examined neglect in different sectors of space, using different tasks, is the study by Vuilleumier et al. (1998). They found a patient that showed neglect for far, but not for near, space in all the tests used (line bisection, reading, cancellation task). However, this does not mean that once a representation is comprosmised by brain damage, then all tasks performed within that space are affected and, vice versa, once a particular space sector is spared, then all tasks are possible in that sector. The performance of a task depends not only on where stimuli are presented in space, but also on the specific cognitive system underlying the task that, if intact, may overcome the spatial problem. Moreover, as suggested by Cowey et al. (1999) and by Vuilleumier et al. (1998), the different motor programs used for performing the tasks may also influence different performances in different tasks. The lesion could affect specific motor programs differently, therefore causing different pattern of results.

The remarkable result of the present experiment is that the use of a stick, by extending the body schema to include the space accessible by the stick, influenced the patient's computation of space. Indeed, when in the far space the patient had to indicate the midpoint of the line by pointing to it, the left part of the line was normally perceived, whereas when the patient had to reach the line by means of the stick, the left part of the line was misperceived, as was in near space. The capacity of using tools is, evolutionary, one of the most important achievement for monkeys and man. By holding a stick, we can reach for objects that are beyond the limit of our arm without using the locomotion. Consequently, the relation between our body and the external objects is modified. A far object can become near if we can reach it, no matter what means we use, the hand or a tool. Iriki et al. (1996) suggested that the bimodal neurons that modify their visual receptive field when the monkey reaches for an object with a stick, the neural correlate of an expanded image of the hand. Based on this hypothesis, and on the assumption that the human brain is equipped in a similar way as the monkey brain, our data can be explained as follows. When the patient used the stick to reach for the object of interest in far space, the tool was coded as part of the patient's hand, as in monkeys, causing an expansion of the representation of the body schema. This affected the spatial relation between far space and the body. The structure of peripersonal space was then altered and peripersonal space was expanded to include the far space reachable by the tool. The reaching of "far" space with a tool determined a switch between spatial representations, so that the representation of near space was now activated. Because near space representation was affected by the brain damaged, the remapping of far space as near affected patient performance in line bisection and neglect reappeared. We did not predict an influence of the use of the projection lightpen in near space, because although the projection lightpen might be incorporated in the representation of the body (like the stick), its dimension is such that it would not alter the relation between personal, peripersonal, and extrapersonal space.

An alternative explanation of our results could be that the kind of action we were going to perform selects the representation of space compatible with that action. According to this alternative hypothesis, our results might be explained as follows: In the space outside the hand-reaching distance, a pointing action activates the representation of far space (intact), whereas a reaching action activates the representation of near space (impaired). This would imply that the simple use of the projection lightpen (pointing action) activates the representation of far space. If so, we should have found a significant amelioration of neglect also in "near" space, when the patient used the projection lightpen. However, we did not find a significant difference between bisection by the index finger (reaching) and bisection by the lightpen (pointing) in near space. Therefore, we are keen to favor the hypothesis stating that the effect that we found in far space, for the reaching modality, is more related to a switch in space representations induced by the artificial extension of body space, than to an activation of different space representations induced by the programming of a specific action. Nonetheless, it is worth noting that both in our patient and in the patient described by Halligan and Marshall (1991) there was a nonsignificant tendency to have a less severe neglect in the pointing modality, also in near space, as if action programming may interact with space mapping.

In conclusion, what affected our patient's performance was the use of a tool that extended the space accessible by the subject influencing the representation of different sectors of space. Both studies on monkeys and neuropsychological studies seem to show that peripersonal and extrapersonal space coding is a dy- 

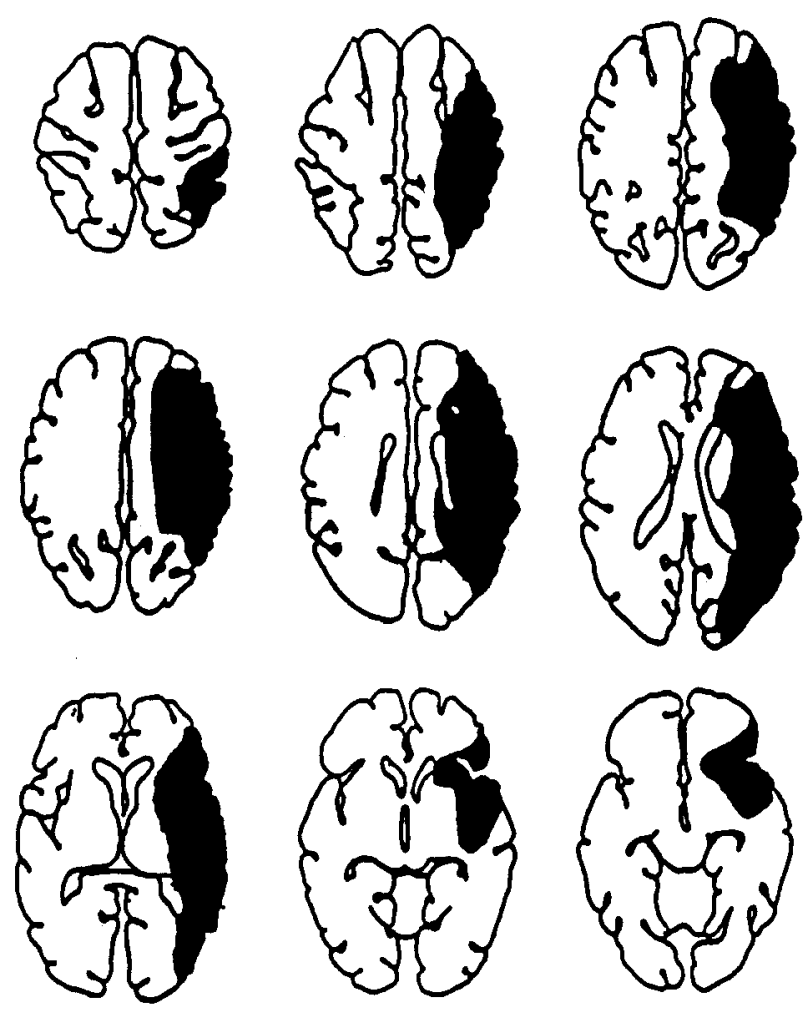

Figure 2. Reconstruction of the patient's brain damage following Damasio and Damasio (1989).

namic process, not only related to the absolute coding of a distance, but also dependent on how body extension is computed in the brain.

\section{METHODS}

P.P. was a 77-year-old, right-handed woman who suffered a right CVA on May 2, 1997. On admission, she showed severe left hemiparesis, left body anesthesia, and left homonymous hemianopia. She was anosognosic for her hemiplegia and her hemianopia, but her general reasoning capacities were within the normal range. A CT scan, performed a month after the stroke, showed an ischemic lesion affecting the lateral aspect of the right frontal, temporal and occipital lobe and the inferior and superior parietal lobe. The right basal ganglia and the insula were also damaged (see Figure 2).

We tested P.P. a month after the stroke (after she had given informed consent to participate in the study) on two different days, with an interval of a week between the two experimental sessions. In both occasions, she showed a severe neglect in the Albert test (Albert, 1973), which requires the patient to cross out 40 lines drawn at various angles and widely dispersed on a sheet of paper. In this test, she cancelled only the rightmost stimuli. Moreover, in reading single words, she omitted the leftmost letters and, in reading a paragraph, she omitted the leftmost words of each line. Therefore, when examined in the near space, P.P. showed neglect for left-side stimuli.

The patient was then tested on line bisection. Lines were drawn in black, on A3 sheets of paper, horizontally oriented, mounted on a wooden board. The board could be placed at a distance of approximately $50 \mathrm{~cm}$ (near space) or at a distance of approximately $100 \mathrm{~cm}$ (far space). The midpoint of each line was positioned at eye-level and aligned with the patient's midline. Line length in the near space could be either $10 \mathrm{~cm}$ (short lines) or $20 \mathrm{~cm}$ (long lines). Line length in the far space was corrected for the visual angle and could be either 20 $\mathrm{cm}$ (short lines) or $40 \mathrm{~cm}$ (long lines). In this way, short lines in near and far space and long lines in near and far space covered the same angle at the retinal level. In the near space the patient had to bisect the line either by touching the midpoint of the line with the index finger of the right hand (reaching modality) or indicating the midpoint of the line by means of a projection lightpen (pointing modality). In the far space, the patient had to bisect the lines either by using a 100-cm stick (reaching modality) or by means of a projection lightpen (pointing modality). Both in the pointing modality and in the reaching modality with the stick, the patient was instructed to keep her right hand close to the body midline. Altogether there were eight conditions: Short and long lines had to be bisected in far or near space, both in the pointing and reaching modality. There were 10 trials for each condition for a total of 80 trials per session.

\section{Acknowledgments}

We are grateful to Giacomo Rizzolatti and Paolo Nichelli for their suggestions and comments on the first draft of the manuscript. We also thank Marzia Minelli for her help in collecting the data and Katie Wright who kindly reviewed the English. This study was supported by a $60 \%$ MURST grant to A.B.

Reprint request should be sent to: Anna Berti, Dipartimento di Psicologia, Università di Torino, Via Lagrange 3, 10123 Turin, Italy.

\section{REFERENCES}

Aglioti, S., Smania, N., Manfredi, M., \& Berlucchi, G. (1996). Disownership of left hand and of objects related to it in a patient with right brain damage. NeuroReport, 8, 293-296.

Albert, M. L. (1973). A simple test for visual neglect. Neurology, 23, 658-664.

Brouchon, N., Joanette, Y., \& Samso, M. (1986). From movement to gesture: "here" and "where" of visually guided pointing. In J. L. Nespoulos, P. Perron, \& A. R. Lecours (Eds.), The biological foundation of gestures: Motor and semiotic aspects (pp. 95-107). London: Erlbaum.

Bruce, C. J., \& Goldberg, M. E. (1985). Primate frontal eye fields. I. Single neurons discharging before saccades. Journal of Neurophysiology, 53, 603-635.

Colby, C. L., \& Duhamel, J. R. (1996). Spatial representation for action in parietal cortex. Cognitive Brain Research, 5, 105. 
Colby, C. L., Duhamel, J. R., \& Goldberg, M. E. (1993). Ventral intraparietal area of the macaque: Anatomic location and visual response properties. Journal of Neurophysiololy, 69, 902-914.

Colby, C. L., Duhamel, J. R., \& Goldberg, M. E. (1996). Visual, presaccadic, and cognitive activation of single neurons in monkey lateral intraparietal area. Journal of Neurophysiology, 76, 2841-2852.

Cowey, A., Small, M., \& Ellis, S. (1994). Left visuo-spatial neglect can be worse in far than in near space. Neuropsychologia, 32, 1059-1066.

Cowey, A., Small, M., \& Ellis, S. (1999). No abrupt change in visual hemineglect from near to far space. Neuropsychologia, 37, 1-6.

Damasio, H., \& Damasio, A. (1989). Lesion analysis in neuropsychology. New York: Oxford University Press.

di Pellegrino, G., Làdavas, E., \& Farnè, A. (1997). Seeing where your hands are. Nature, 338, 730.

Duhamel, J. R., Bremmer, F., BenHamed, S., \& Graf, W. (1997). Spatial invariance of visual receptive filed in parietal cortex neurons. Nature, 389, 845-848.

Fogassi, L., Gallese, V., Fadiga, L., Luppino, G., Matelli, M., \& Rizzolatti, G. (1996). Coding of peripersonal space in inferior premotor cortex (area F4). Journal of Neurophysiology, 76, $141-157$.

Gentilucci, M., Fogassi, L., Luppino, G., Matelli, M., Camarda, R., Rizzolatti, G. (1988). Functional organization of inferior area 6 in the macaque monkey. I. Somatotopy and the control of proximal movements. Experimental Brain Research, 71, 475-490.

Goldberg, M. E., \& Bushnell, M. C. (1981). Behavioral enhancement of visual responses in monkey cerebral cortex. II. Modulation in frontal eye field specifically related to saccades. Journal of Neurophysiology, 46, 773-787.

Graziano, M. S. A., \& Gross, C. G. (1995). The representation of extrapersonal space: Possible role for bimodal, visual-tactile neurons. In M. Gazzaniga (Ed.), The cognitive neuroscience (pp. 1021-1033). Cambridge: MIT Press.

Graziano, M. S. A., Yap, G. S., \& Gross, C. G. (1994). Coding of visual space by premotor neurons. Science, 266, 1054.

Halligan, P., \& Marshall, J. M. (1991). Left neglect for near but not for far space in man. Nature, 350, 498-500.

Head, H., \& Holmes, G. (1911). Sensory disturbances from cerebral lesion. Brain, 34, 102-254.
Iriki, A., Tanaka, M., \& Iwamura, Y. (1996). Coding of modified body schema during tool use by macaque postcentral neurons. NeuroReport, 7, 2325-2330.

Làdavas, E., di Pellegrino, G., Fernè, A., \& Zeloni, G. (1998). Neuropsychological evidence of an integrated visuotactile representation of peripersonal space in humans. Journal of Cognitive Neuroscience, 10, 1-24.

Làdavas, E., Zeloni, G., Farnè, A. (1999). Visual peripersonal space centered on the face in humans. Brain, 421, 23172329.

Latto, R., \& Cowey, A. (1971). Visual field defects after frontal eye-field lesions in monkeys. Brain Research, 30, 1-24.

Leinonen, L., Hyvarinen, G., Nymani, G., Linnankoski, I. (1979). I. Functional properties of neurons in lateral part of associative area 7 in awake monkeys. Experimental Brain Research, 34, 299.

Paillard, J. (1993). In A. Berthelet, \& J. Chavaillon (Eds.), The use of tools by human and non-human primates (pp. 3646). New York: Oxford University Press.

Rizzolatti, G., \& Gallese, V. (1988). Mechanisms and theories of spatial neglect. In F. Boller, \& J. Grafman (Eds.), Handbook of neuropsychology, Vol. 1 (pp. 223-249). Amsterdam, the Netherlands: Elsevier.

Rizzolatti, G., Gentilucci, M., \& Matelli, M. (1985). Selective spatial attention: One center, one circuit or many circuits? In M. I. Posner, \& O. M. Marin (Eds.), Attention and performance, Vol. XI (pp. 251-265). Hillsdale, NJ: Erlbaum.

Rizzolatti, G., Matelli, M., \& Pavesi, G. (1983). Deficits in attention and movement following the removal of postarcuate (area 6) cortex in macaque monkeys. Brain, 106, 655-673.

Rizzolatti, G., Riggio, L., \& Sheliga, B. M. (1994). Space and selective attention. In C. Umilta, \& M. Moscovitch (Eds.), Attention and performance (pp. 231-265). Cambridge: MIT Press.

Shelton, P. A., Bowers, D., \& Heilman, K. M. (1990). Peripersonal and vertical neglect. Brain, 113, 191-205.

Thier, P., \& Andersen, R. A., (1997). Multiple parietal 'eye field': Insight from electrical microstimulation. In P. Thier, \& H. O. Karnath (Eds.), Parietal lobe contributions to orientation in 3D space (pp. 95-108). Heidelberg: Springer Verlag.

Vuilleumier, P., Valenza, N., Mayer, E., Reverdin, A., \& Landis, T. (1998). Near and far visual space in unilateral neglect. Annals of Neurology, 43, 406-410. 